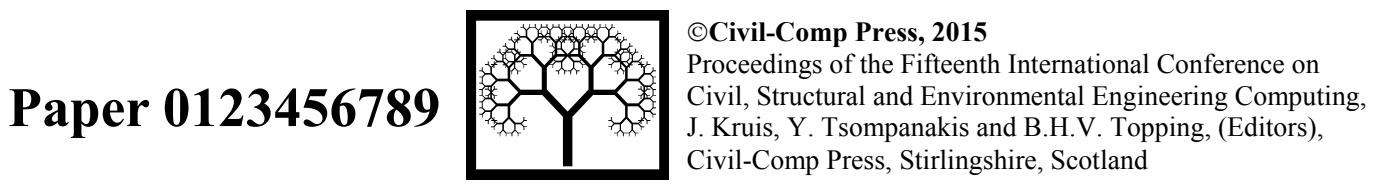

\title{
A Formulation for Hyperelastic Damaged Materials
}

\author{
M. Miñano and F.J. Montáns \\ Escuela Técnica Superior de Ingeniería Aeronáutica y del Espacio \\ Universidad Politécnica de Madrid, Spain
}

\begin{abstract}
Hyperelastic materials are characterized by an energy-preserving behavior which results in an identical path for loading, unloading and reloading. Rubbery materials consist frequently on a cross-linked elastomeric substance containing some percentage of particles of carbon as fillers. Due to such fillers, for example, the virgin loading path differs substantially from the unloading-reloading one. The behavior may then be considered as a result of damage in the material. This effect is known as part of the Mullins effect and is present not only in carbon-filled rubbers but also in biological materials.

In this paper we present a novel formulation of continuum damage mechanics in hyperelastic materials and an efficient computational procedure for modelling the Mullins effect in isochoric, isotropic materials. The formulation is based on the idea that undamaged hyperelastic behavior cannot be measured, but the unloadingreloading behavior of a damaged material can be readily obtained and on that only the unloading-reloading curve presents a real hyperelastic behavior. The unloadingreloading curve may be described by any appropriate constitutive model but using spline-based functions both the virgin loading and the unloading-reloading curves are exactly captured. The model is efficient for finite element implementation.
\end{abstract}

Keywords: hyperelasticity, logarithmic strains, Mullins effect, damage mechanics, living tissues, polymers.

\section{Introduction}

Many materials like biological tissues and rubbers are usually modelled as hyperelastic isochoric materials $[1,2,3,4,5]$. Hyperelastic materials preserve the energy during closed cycles. Nevertheless many of them display a dissipative behavior known 
as Mullins effect $[6,7,8,9,10,11,12]$ which is frequently modelled as without permanent strains. There is as yet no widespread agreement on the physical phenomena involved whose consequence is a reduction of stiffness $[12,13,14,15,16,17,18]$ from a phenomenological point of view. In practice, several other inelastic effects arise under loading and unloading cycles as residual strains [19, 20], dependence of the shape of the stress-strain curves with rate and temperature, induced anisotropy, among others. The simplest approach is to model the effect as an isotropic softening effect through continuum-based damage models [2, 5, 12, 14, 17, 21, 22, 23, 24]. The most widely used approach for the case of isotropic damage is to suppose an undamaged stored energy function and then apply the reduction factor $(1-D)$ where $D$ $\in[0,1)$ is the scalar damage variable, first proposed by Kachanov [25]. Actually, and this is a main point of this paper, the undamaged stored energy function can not be measured, but only the damaged one. Additionally a damage criterion and an evolution function $[2,26,27]$ are usually established for the damage variable. Finally, a parameter-fitting procedure is used to obtain the material constants of the damage hyperelastic model.

We propose a different approach in which using spline-based hyperelastic models we are able to capture exactly the experimental stress-strain curves without using any fitting material parameter $[28,29]$. Then a new formulation is developed in which the unloading-reloading hyperelastic curves are directly obtained from the experimental data.

In the following sections, we first present the continuum constitutive equations for isochoric, isotropic damage following a new approach similar to the split operator typical of computational plasticity. Then we introduce the damage scalar variable. Finally we give some illustrative examples.

\section{Theoretical background}

Let $\boldsymbol{E}$ be the logarithmic strains in the reference configuration and $\boldsymbol{T}$ the generalized Kirchoff stress, work-conjugate of the logarithmic stress [30]. Let $w_{D}$ be an internal variable and $\mathcal{W}\left(\boldsymbol{E}, w_{D}\right)$ the stored energy per unit reference volume. The conservation of energy states that

$$
\dot{\mathcal{W}}=\boldsymbol{T}: \dot{\boldsymbol{E}}=\frac{\mathcal{W}\left(\boldsymbol{E}, w_{D}\right)}{\partial \boldsymbol{E}}: \dot{\boldsymbol{E}}+\frac{\partial \mathcal{W}\left(\boldsymbol{E}, w_{D}\right)}{\partial w_{D}} \frac{\partial w_{D}}{\partial \boldsymbol{E}}: \dot{\boldsymbol{E}}
$$

then,

$$
\boldsymbol{T}=\frac{d \mathcal{W}(\boldsymbol{E})}{d \boldsymbol{E}}=\left.\frac{\mathcal{W}\left(\boldsymbol{E}, w_{D}\right)}{\partial \boldsymbol{E}}\right|_{\dot{w}_{D}=0}+\left.\frac{\partial \mathcal{W}\left(\boldsymbol{E}, w_{D}\right)}{\partial w_{D}}\right|_{\dot{\boldsymbol{E}}=0} \frac{\partial w_{D}}{\partial \boldsymbol{E}}
$$

The differentiation in Equation (2) can be construed as a trial-hyperelastic stress predictor and a damage-stress-corrector parts which is a typical approach employed in computational elastoplasticity and viscoleasticity. However, as a remarkable difference with computational plasticity and viscoelasticity, the trial and final states correspond to the same strain. 
The uncoupled form of the stored energy function is given by

$$
\mathcal{W}=\mathcal{U}(J)+\mathcal{W}^{d}\left(\boldsymbol{E}^{d}, w_{D}\right)
$$

For the isotropic case, the Valanis-Landel hypothesis states for a fixed value of $w_{D}$

$$
\overline{\mathcal{W}}^{d}\left(\lambda_{1}^{d}, \lambda_{2}^{d}, \lambda_{3}^{d}, w_{D}\right)=\bar{\omega}\left(\lambda_{1}^{d}, w_{D}\right)+\bar{\omega}\left(\lambda_{2}^{d}, w_{D}\right)+\bar{\omega}\left(\lambda_{3}^{d}, w_{D}\right)
$$

Usually damage is considered to affect only the isochoric contribution and to not affect incompressibility, so

$$
\mathcal{W}^{d}\left(\ln \lambda_{1}, \ln \lambda_{2}, \ln \lambda_{3}, w_{D}\right)=\omega\left(E_{1}, w_{D}\right)+\omega\left(E_{2}, w_{D}\right)+\omega\left(E_{3}, w_{D}\right)
$$

We define the damage variable as the maximum isochoric energy reached up to time $\mathrm{t}$

$$
w_{D}=\max _{\tau \in(-\infty, t)}\left(\mathcal{W}^{d}(\tau)\right)
$$

A similar damage variable has been introduced by several authors, see for example $[22,27,31]$. For different level of damage we have

$$
\mathcal{W}^{d}\left(\boldsymbol{E}^{d}, w_{D 1}\right), \mathcal{W}^{d}\left(\boldsymbol{E}^{d}, w_{D 2}\right), \ldots
$$

and during unloading-reloading without further damage

$$
\mathcal{W}_{D 1}^{d}\left(\boldsymbol{E}^{d}\right), \mathcal{W}_{D 2}^{d}\left(\boldsymbol{E}^{d}\right)
$$

The functions of the Equation (8) are true hyperelastic functions, however the Equation (4) is a potential function which depends on damage (dissipative variable). Each energy function of Equation (8) can be written using the Valanis-Landel decomposition as

$$
\mathcal{W}_{D n}^{d}\left(\boldsymbol{E}^{d}\right)=\omega_{D n}\left(E_{1}^{d}\right)+\omega_{D n}\left(E_{2}^{d}\right)+\omega_{D n}\left(E_{3}^{d}\right)
$$

Experimental evidences show that damage decreases the stored energy. We define the energy release rate tensor as

$$
\boldsymbol{Y}=-\frac{\partial \mathcal{W}^{d}\left(\boldsymbol{E}^{d}, w_{D}\right)}{\partial w_{D}} \frac{\partial w_{D}}{\partial \boldsymbol{E}}=\gamma \boldsymbol{D}
$$

where $\gamma=-\partial \mathcal{W}^{d}\left(\boldsymbol{E}^{d}, w_{D}\right) / \partial w_{D}$ is the scalar damage power loss factor.

The stored energy rate results

$$
\begin{aligned}
\dot{\mathcal{W}} & =\dot{\mathcal{U}}(J)+\dot{\mathcal{W}}^{d}\left(\boldsymbol{E}^{d}, w_{D}\right) \\
& =\frac{\partial \mathcal{U}(J)}{\partial \boldsymbol{E}}: \dot{\boldsymbol{E}}+\frac{\partial \mathcal{W}_{D}^{d}\left(\boldsymbol{E}^{d}\right)}{\partial \boldsymbol{E}^{d}}: \frac{\partial \boldsymbol{E}^{d}}{\partial \boldsymbol{E}}: \dot{\boldsymbol{E}}+\underbrace{\frac{\partial \mathcal{W}^{d}\left(\boldsymbol{E}^{d}, w_{D}\right)}{\partial w_{D}}}_{\leq 0} \underbrace{\frac{\partial w_{D}}{\partial \boldsymbol{E}}: \dot{\boldsymbol{E}}}_{\geq 0} \\
& =\boldsymbol{T}^{v}: \dot{\boldsymbol{E}}+\boldsymbol{T}^{d}: \dot{\boldsymbol{E}}
\end{aligned}
$$




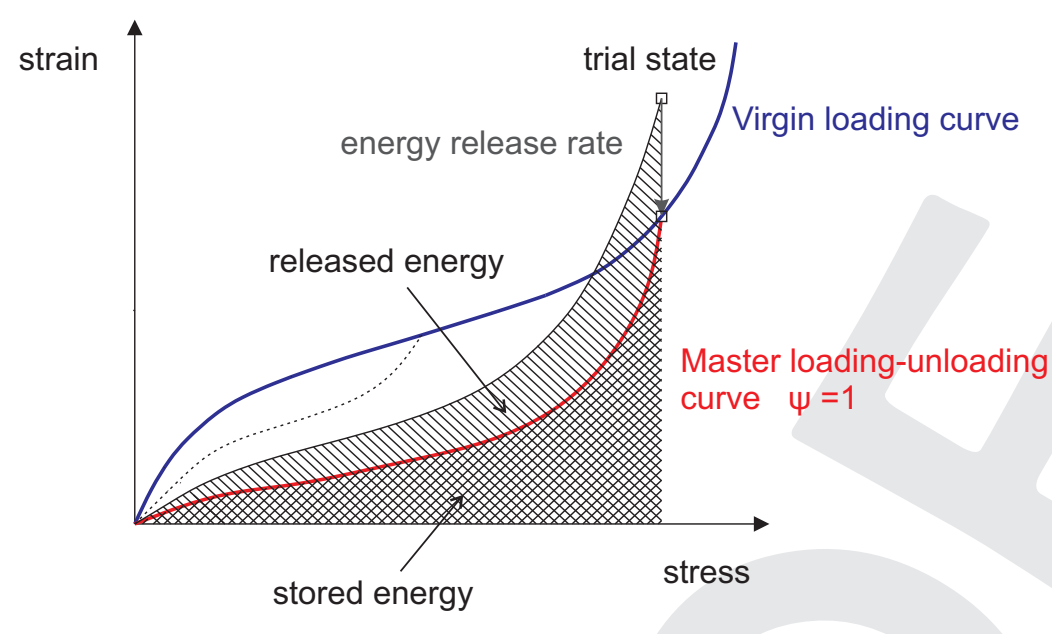

Figure 1: Representation of the the virgin loading and master loading-unloading curves, of the stored and released energies and definition of the trial state.

where ${ }^{t r} \boldsymbol{T}^{d}$ is defined as the trial isochoric Kirchhoff stress tensor, obtained with the damage frozen, $\boldsymbol{Y}$ is the correction due to damage and $\boldsymbol{T}^{d}$ is the resulting isochoric Kirchhoff stress tensor. Finally this leads to

$$
\left\{\begin{array}{l}
\boldsymbol{T}^{d}={ }^{t r} \boldsymbol{T}^{d} \text { for no damage increase; } \dot{w}_{D}=0 \\
\boldsymbol{T}^{d}={ }^{t r} \boldsymbol{T}^{d}-\boldsymbol{Y} \text { for damage increase; } \dot{w}_{D}>0
\end{array}\right.
$$

A representation of all these terms is given in Figure 1.

According to the damage variable introduced, we establish the following damage criterion

$$
f_{D}=\mathcal{W}^{d}-w_{D} \leq 0 \Longleftrightarrow \mathcal{W}^{d} \leq w_{D}
$$

The consistency condition during damage process states that

$$
\dot{f}_{D}=\left({ }^{t r} \boldsymbol{T}^{d}-\boldsymbol{Y}-\frac{\partial w_{D}}{\partial \boldsymbol{E}}\right): \dot{\boldsymbol{E}}=0 \text { for } \dot{w}_{D}>0
$$

Considering that the evolution of damage can only occur when there is increasing deformation $\dot{\boldsymbol{E}} \neq 0$

$$
\boldsymbol{D}:=\frac{\partial w_{D}}{\partial \boldsymbol{E}}={ }^{t r} \boldsymbol{T}^{d}-\boldsymbol{Y}=\boldsymbol{T}^{d}
$$

From Equation (10)

$$
\boldsymbol{Y}=\frac{\gamma}{1+\gamma}{ }^{t r} \boldsymbol{T}^{d} \Rightarrow \boldsymbol{T}^{d}=\frac{1}{1+\gamma}{ }^{t r} \boldsymbol{T}^{d}
$$

Compared with computational elastoplasticity, the Equation (17) is basically similar to the radial return algorithm of Wilkins [32]. 
It can be shown [33] that the Valanis-Landel decomposition results into a expression of the type

$$
\mathcal{W}^{d}\left(E_{1}, E_{2}, E_{3}, w_{D}\right)=\psi\left(w_{D}\right) \mathcal{W}_{0}^{d}\left(E_{1}, E_{2}, E_{3}\right)
$$

where $\mathcal{W}_{0}^{d}$ is a reference, largely damaged, stored energy

$$
\mathcal{W}_{0}^{d}\left(E_{1}^{d}, E_{2}^{d}, E_{3}^{d}\right)=\omega_{0}\left(E_{1}^{d}\right)+\omega_{0}\left(E_{2}^{d}\right)+\omega_{0}\left(E_{3}^{d}\right)
$$

The factor of proportionality $\psi\left(w_{D}\right)$ is obviously different for each level of damage.

In summary, only two scalar functions, which can be obtained from a tensile loadingunloading test, are required. The energy $\mathcal{W}_{0}^{d}$ can be obtained from the user-prescribed spline-based stored energy function for the maximum expected strain. The factor of proportionality $\psi\left(w_{D}\right)$ is determined as the quotient between the stress given by the uniaxial virgin test $T$ and the stress given by the master hyperelastic curve $T_{0}$.

$$
\psi\left(w_{D}(E)\right)=\frac{T}{T_{0}}
$$

Equation (18) is similar to that of classical damage formulations [2], nevertheless it should be noted that in our formulation it is a result of isotropy and Valanis-Landel decomposition. It is noteworthy that the undamaged energy used in most of the models $[2,22,27,34]$ cannot be obtained experimentally whereas the damaged one $\mathcal{W}_{0}^{d}$ can be readily determined. In addition, the virgin loading curve cannot be obtained from a hyperelastic curve because itself involves damage which is a dissipative process.

\section{Examples}

We give in this section some illustrative examples of the model. The examples are selected, with assumed test data, simply to show the capabilities of the model. The material data needed for the model is merely an experimental virgin loading curve and the master damaged unloading-reloading curve. This master unloading-reloading curve must be given up to the maximum expected strain. We have interpolated both experimental curves using piece-wise splines in order to obtain analytical, continuous functions. The original data employed and the resulting uniform spline interpolation are shown in Figure 2. The material is modeled as quasi-incompressible. Therefore, we introduced a penalty volumetric function with a large equivalent bulk modulus in order to numerically enforce incompressibility when required. No additional information is needed for the model.

As a first simulation we have imposed the uniaxial strain history $E_{1}$ shown in Figure 3a. As a result of incompressibility $E_{2}=E_{3}=-0.5 E_{1}$. As shown in Figure 3c, the predicted stress-strain data exactly simulate the prescribed data. Note that we represent $T_{1}-T_{2}$ to remove the indeterminate pressure. In Figure $3 \mathrm{~b}$ it can be seen the resulting damage variable evolution versus the strain. Finally in Figure $3 \mathrm{~d}$ we illustrate the evolution of the reference isochoric stored energy during the simulation. 


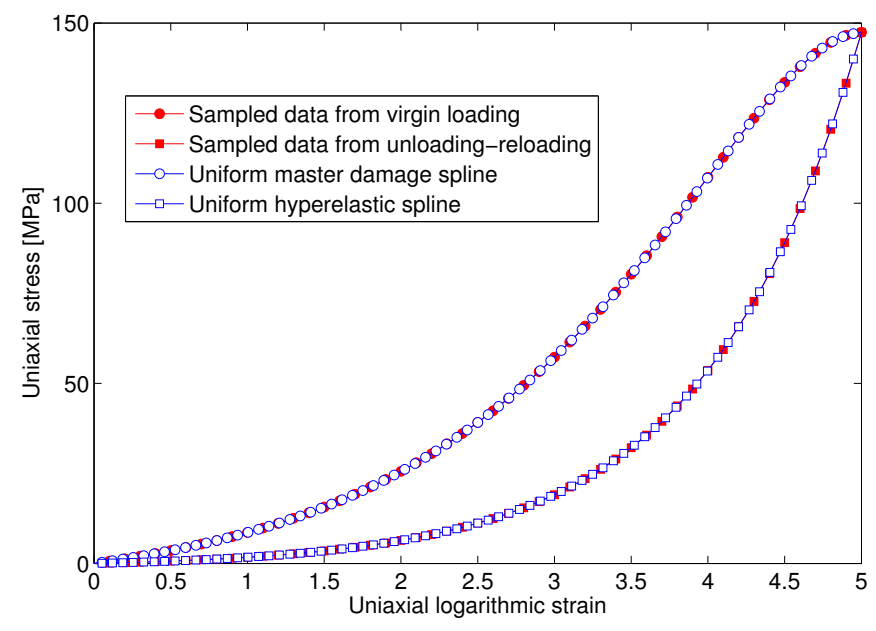

Figure 2: Original stress-strain data and spline fit.

As a second simulation, we prescribe a simple shear history which deformation gradient is

$$
{ }_{0}^{t} \boldsymbol{X}=\left[\begin{array}{ccc}
1 & \gamma(t) & \\
& 1 & \\
& & 1
\end{array}\right]
$$

where $\gamma(t)$ is the shearing parameter. Notice that the imposed deformations are isochoric. In Figure $4 \mathrm{a}$ is shown the shear history. The resulting strains are shown in Figure $4 \mathrm{~b}$. The predictions using the model are shown in Figure $4 \mathrm{c}$ and Figure 4d. We note that the shear stress reaches a maximum depending on the nonlinearity of the stored energy with the logarithmic shear strain. This is an observation inherent to the simple shear test at large strains and which is physically sound [30]. Logarithmic strain measures simply reveal this fact. We can observe that for large strains the components $T_{11}$ and $T_{22}$ reach values much larger than shear stresses [30].

The evolution during the simulation of the damage multiplier $\psi$ and of the damage variable $w_{D}$ are given in Figure 5.

As final simulation, in order to evaluate the capabilities of the model we have prescribed a combined nonproportional axial-torsion load test. This test has been stressdriven. Figure 6a shows the prescribe stresses where $T_{22}=T_{33}=T_{13}=T_{23}=0$. The resulting strains are given in Figure $6 \mathrm{~b}$.

Figure 7 represents the evolution of the reference isochoric energy $W_{0}^{d}$ and of the damage variable $w_{D}$.

In the table of Figure 8 we represent the numerical performance of the algorithm. We have used plain Newton algorithms both locally and globally. We have selected two global step, one in which there is damage evolution and another step where no damage evolution occurs. The local column shows the number of local iterations used in obtaining the energy multiplier parameter $\psi$ until the consistency condition is sat- 


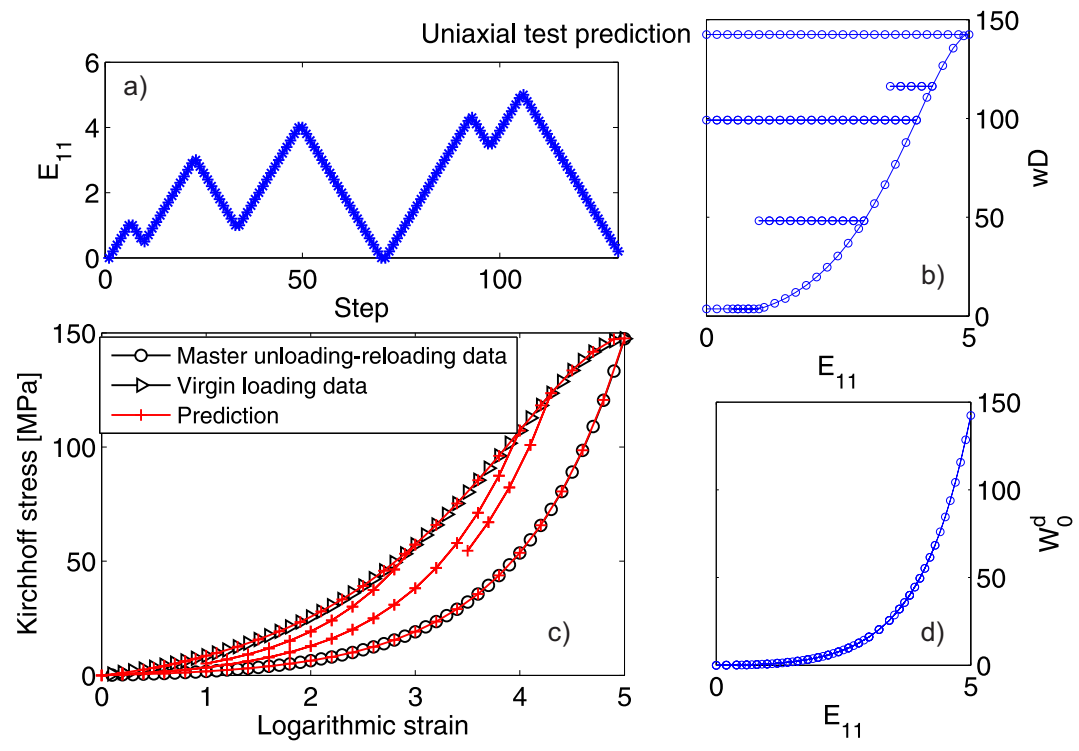

Figure 3: Predictions for the uniaxial test. a)Prescribed uniaxial strain. b) Evolution of the damage variable during the simulation. c) Predictions and uniaxial prescribed data. d) Evolution of the reference isochoric stored energy during the simulation.

isfied with a relative tolerance of the damage function of $10^{-10}$. For this example, the typical number of iterations employed per global iteration is 3 . The global column shows the number of global equilibrium iterations required in each step using a consistent tangent [33]. Usually it employs 4-5 iterations per step for a relative tolerance of the loads of $10^{-10}$.

We note that the computational algorithm may be consistently linearized and if so, second order convergence is achieved. In fact, note that iterations are due to the hyperelastic response except during damage evolution [33].

\section{Conclusions}

In this work we present a new formulation and computational procedure for damage in isotropic, isochoric hyperelastic materials. The approach is based on the idea that is not possible to measure the undamaged stored energy function, but only the damaged one. The continuum formulation is based on an operator split which can be interpreted as a trial-hyperelastic stress predictor and a damage-stress-corrector. Employing spline-based stored energy functions, we are capable of replicating both the virgin loading and the unloading-reloading behavior. We have shown that the algorithm is very efficient per finite element simulations. 

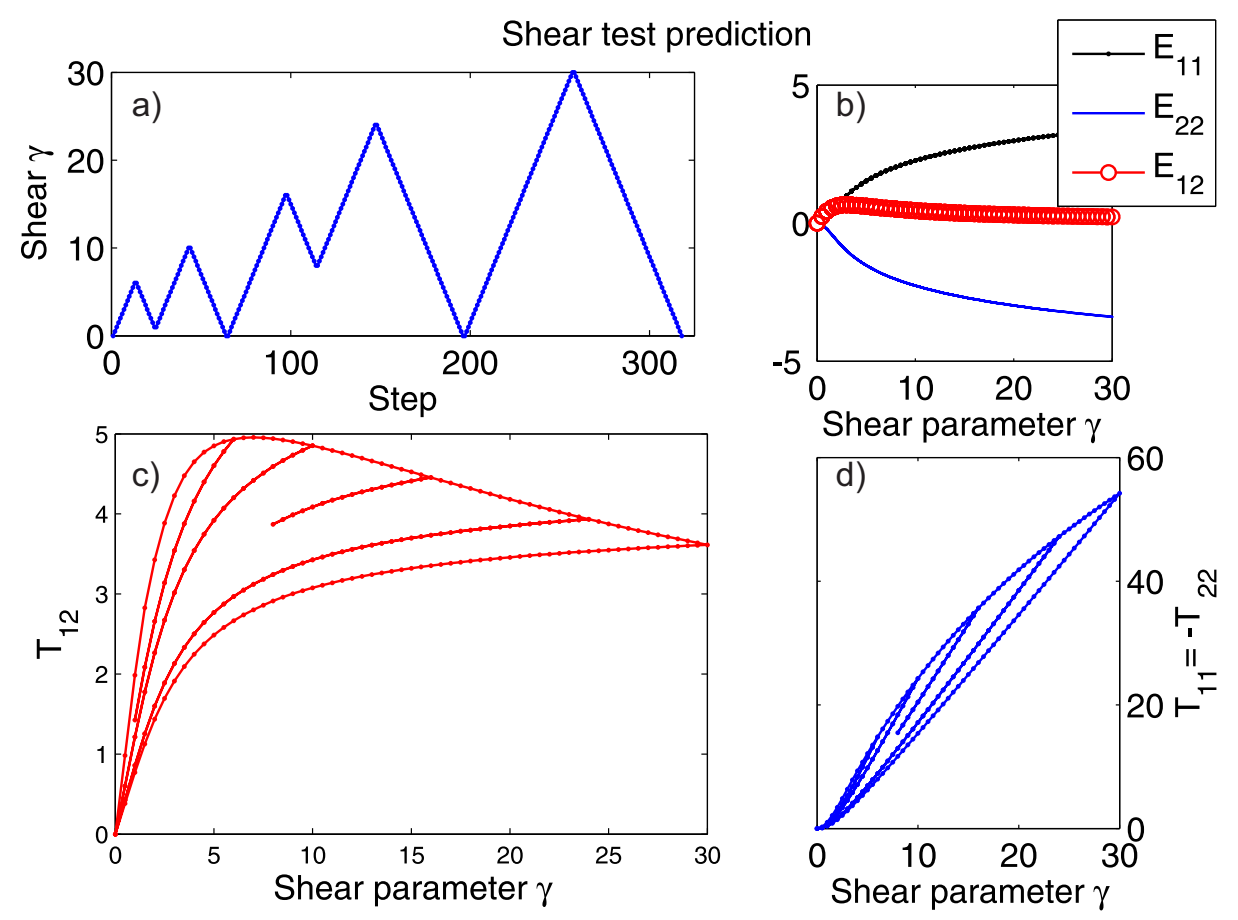

Figure 4: Simple shear test prediction. a) The time history for the shear parameter. b) Resulting strains computed from the deformation gradient. c) Predictions for the $T_{12}$ stress versus the shear parameter. d) Predictions for the componentes $T_{11}$ and $T_{22}$.

\section{Acknowledgements}

Partial financial support for this work has been given by grant DPI2011-26635 from the Dirección General de Proyectos de Investigación of the Ministerio de Economía y Competitividad of Spain.

\section{References}

[1] R.W. Ogden, "Non-linear elastic deformations", Dover, New York, 1997.

[2] G.A. Holzapfel, "Nonlinear Solid Mechanics: A Continuum Approach for Engineering", Wiley, Chichester, 2000.

[3] M.T. Shaw, W.J. MacKnigth, "Introduction to Polymer Viscoelasticity", John Wiley \& Sons., 2005.

[4] A.S. Argon, "The Physics of Deformation and Fracture of Polymers", Cambridge University Press, 2013.

[5] J.D. Humphrey, "Cardiovascular Solid Mechanics: Cells, Tissues, and Organs", Springer Science \& Business Media, 2002. 

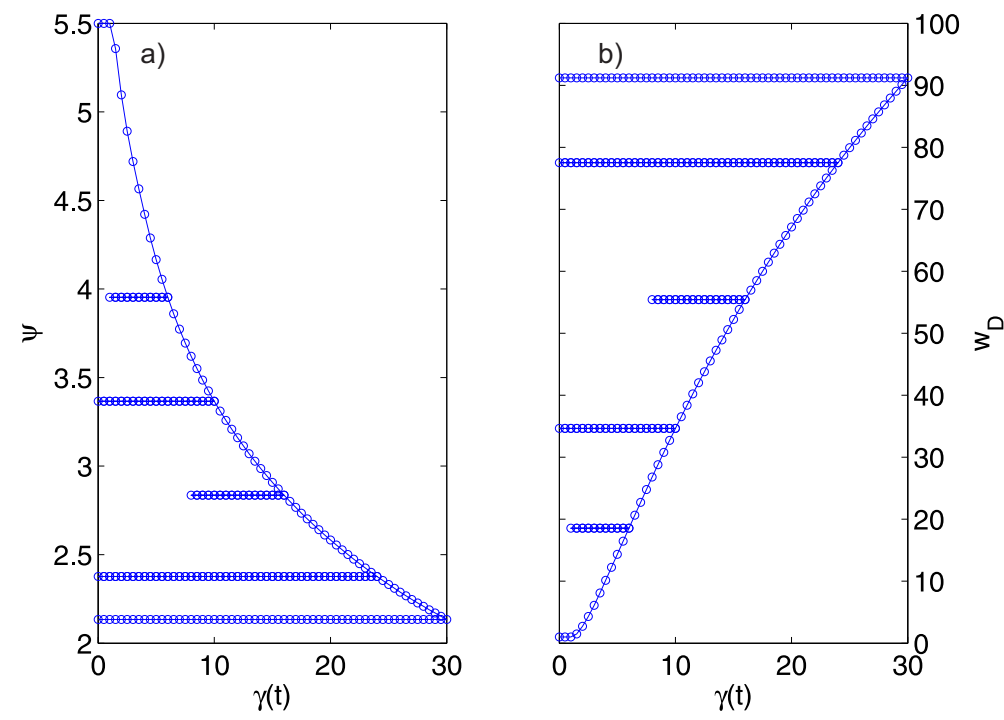

Figure 5: a) Evolution of the damage multiplier during the simple shear test. b) Evolution of the damage variable during the simulation.

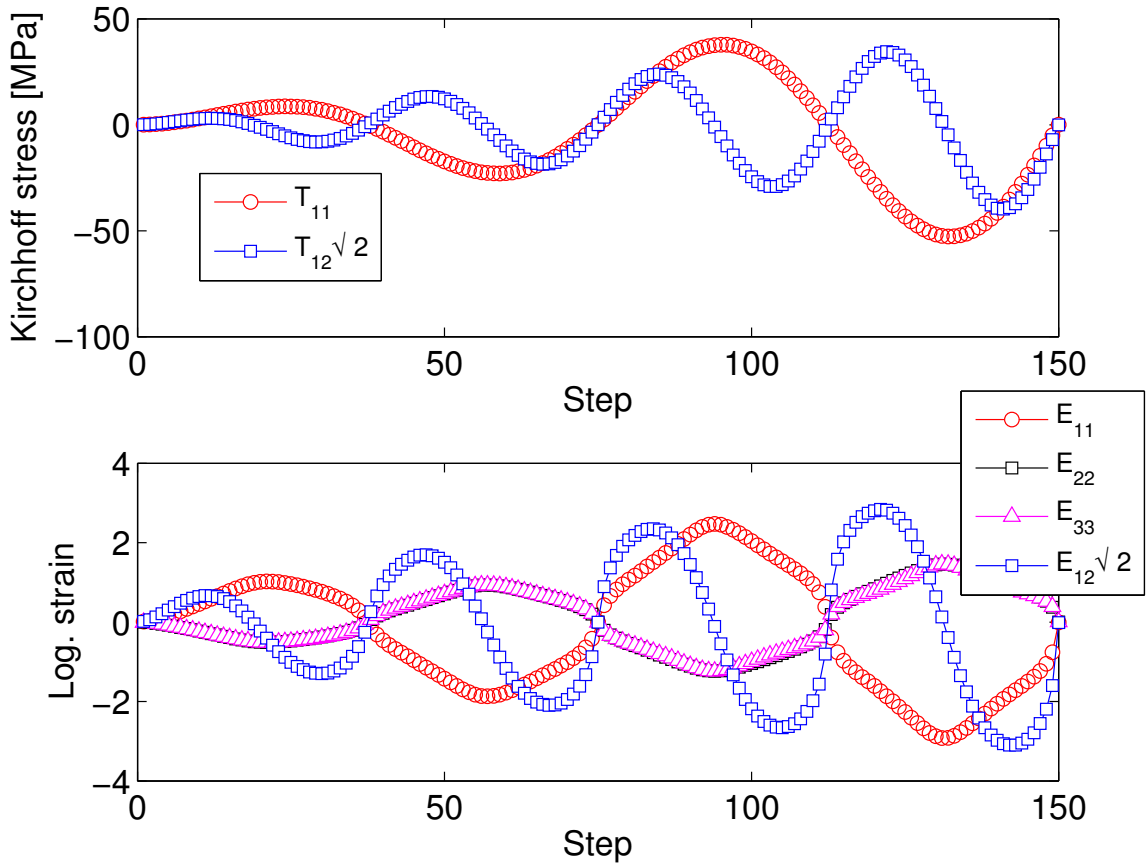

Figure 6: Axial-torsion test. a) History of the imposed axial stress $T_{11}$ and shear stress $T_{12}$. b) Predictions for the strain components $E_{11}, E_{22}, E_{33}$ and $E_{12}$. 

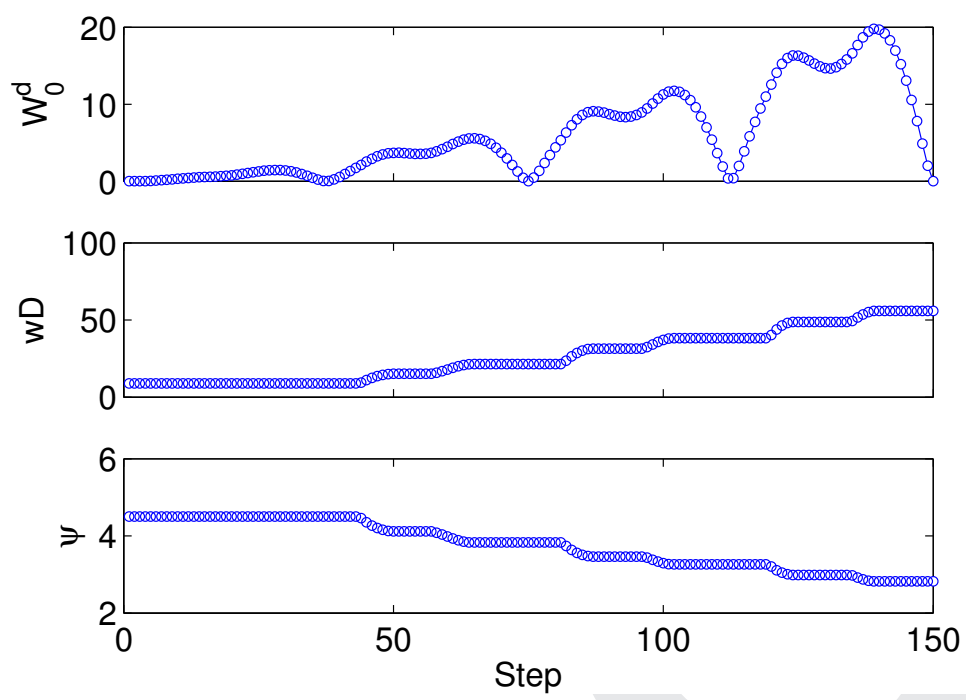

Figure 7: Axial-torsion test. a) Evolution of the reference isochoric energy. b) Evolution of the damage variable.

\begin{tabular}{|c|c|cc|c|}
\hline \multicolumn{2}{|c|}{ Without damage evolution } & \multicolumn{3}{c|}{ With damage evolution } \\
\hline \multicolumn{2}{|c|}{ step 25 } & \multicolumn{3}{c|}{ step 50 } \\
\hline Global & Local & \multicolumn{2}{c|}{ Global } & Local \\
\hline$|\mathrm{R}|$ & $\mathrm{f}$ & \multicolumn{2}{|c|}{$|\mathrm{R}|$} & $\mathrm{f}$ \\
\hline 0.89369 & no iterations & iter 1 & 1.4334 & -0.0012305 \\
0.038785 & & & & $-2.2898 \mathrm{E}-8$ \\
$6.3933 \mathrm{E}-5$ & & & & $-4.9738 \mathrm{E}-14$ \\
$2.8088 \mathrm{E}-10$ & & iter 2 & 0.0093261 & $\ldots$ \\
& & iter 3 & 0.00039882 & $\ldots$ \\
& & iter 4 & $1.0647 \mathrm{E}-7$ & -0.0013013 \\
& & & & $-2.5595 \mathrm{E}-8$ \\
& & & & $4.4409 \mathrm{E}-14$ \\
& & iter 5 & $5.9021 \mathrm{E}-11$ & \\
\hline
\end{tabular}

Figure 8: Axial-torsion test. Number of local and global iterations employed when there is damage evolution and when no damage evolution occurs. 
[6] L. Mullins, "Effect of stretching on the properties of rubber", Rubber Chemistry and Technology 21(2), 281-300, 1948.

[7] L. Mullins, N. R. Tobin, "Theoretical model for the elastic behavior of fillerreinforced vulcanized rubbers", Rubber Chemistry and Technology 30(2), 555$571,1957$.

[8] L. Mullins, "Softening of rubber by deformation", Rubber chemistry and Technology, 42(1), 339-362, 1969.

[9] F. Bueche, "Molecular basis for the Mullins effect", Journal of Applied Polymer Science, 4(10), 107-114, 1960.

[10] M.A. Johnson, M.F. Beatty, "The Mullins effect in uniaxial extension and its influence on the transverse vibration of a rubber string", Continuum Mechanics and Thermodynamics 5(2), 83-115, 1993.

[11] M.A. Johnson, M.F. Beatty, "A constitutive equation for the Mullins effect in stress controlled uniaxial extension experiments." Continuum Mechanics and Thermodynamics 5(4), 301-318, 1993.

[12] J. Diani, B. Fayolle, P. Gilormini, "A review on the Mullins effect", European Polymer Journal, 45(3), 601-612, 2009.

[13] M.A. Johnson, M.F. Beatty, "A constitutive equation for the Mullins effect in stress controlled uniaxial extension experiments", Continuum Mechanics and Thermodynamics 5(4), 301-318, 1993.

[14] R.W. Ogden, D.G. Roxburgh, "A pseudo-elastic model for the Mullins effect in filled rubber", Proceedings of the Royal Society of London, Series A: Mathematical, Physical and Engineering Sciences, 455, 2861-2877, 1999.

[15] A. Dorfmann, R.W. Ogden, "A pseudo-elastic model for loading, partial unloading and reloading of particle-reinforced rubber", International Journal of Solids and Structures 40(11), 2699-2714, 2003.

[16] A. Dorfmann, R.W. Ogden, " A constitutive model for the Mullins effect with permanent set in particle-reinforced rubber. International Journal of Solids and Structures, 41(7), 1855-1878, 2004.

[17] S. Govindjee, J.C. Simo, "A micro-mechanically based continuum damage model for carbon black-filled rubbers incorporating Mullins' effect", Journal of the Mechanics and Physics of Solids 39(1), 87-112, 1991.

[18] M. Kaliske, H. Rothert, "Constitutive approach to rate-independent properties of filled elastomers", International Journal of Solids and Structures 35(17), 20572071, 1998.

[19] G.A. Holzapfel, M. Stadler, R.W. Ogden, "Aspects of stress softening in filled rubbers incorporating residual strains", Proceedings of the First European Conference on Constitutive Models for Rubber, 189-193, Rotterdam, 1999.

[20] R.W. Ogden, D.G. Roxburgh, "An energy-based model of the Mullins effect", Constitutive Models for Rubber, 23-28(6), Balkema, Rotterdam, 1999.

[21] S. Govindjee, J.C. Simó, "Mullins effect and the strain amplitude dependence of the storage modulus", International journal of solids and structures 29(14), 1737-1751, 1992.

[22] J.C. Simo, "On a fully three-dimensional finite-strain viscoelastic damage 
model: formulation and computational aspects", Computer methods in applied mechanics and engineering, 60(2), 153-173, 1987.

[23] M. Miñano, F.J. Montans, "Engineering damage mechanics review", Computational Technology Reviews, v.10, Ch.1, Topping BHV Ed. Saxe-Coburg publications, 2014.

[24] E.A. de Souza Neto, D. Peric, D.R.J. Owen, "Computational methods for plasticity: theory and applications", John Wiley \& Sons, 2011.

[25] L.M. Kachanov, "Time of the rupture process under creep conditions", Izvestija Akademii Nauk Sojuza Sovetskich Socialisticeskich Respubliki (SSSR) Otdelenie Techniceskich Nauk (Moskra) 8, 26-31, 1958.

[26] M. Doblaré, "An anisotropic pseudo-elastic approach for modelling Mullins effect in fibrous biological materials", Mechanics Research Communications 36(7), 784-790, 2009.

[27] E. Peña, B. Calvo, M.A. Martínez, M. Doblaré, "On finite-strain damage of viscoelastic-fibred materials. Application to soft biological tissues", International Journal for Numerical Methods in Engineering 74(7), 1198-1218, 2008.

[28] T. Sussman, K.J. Bathe, "A model of incompressible isotropic hyperelastic material behavior using spline interpolations of tension-compression test data", Communications in numerical methods in engineering 25(1), 53-63, 2009.

[29] M. Latorre, F.J. Montáns, "Extension of the Sussman-Bathe spline-based hyperelastic model to incompressible transversely isotropic materials", Computers and Structures, 122, 13-26, 2013.

[30] M. Latorre, F.J. Montáns, ”On the interpretation of the logarithmic strain tensor in an arbitrary system of representation", International Journal of Solids and Structures 51(7), 1507-1515, 2014.

[31] B. Calvo, E. Peña, M.A. Martínez, M. Doblaré,"An uncoupled directional damage model for fibred biological soft tissues. Formulation and computational aspects", International Journal for Numerical Methods in Engineering 69(10), 2036-2057, 2008.

[32] J.C. Simo, T.J.R. Hughes, ”Computational inelasticity", Springer, 2008.

[33] M. Miñano, F.J. Montans, "A new approach to modeling isotropic damage for Mullins effect in hyperelastic materials",under review.

[34] E.A. de Souza Neto, D. Perić, D.R.J. Owen, "A phenomenological threedimensional rate-idependent continuum damage model for highly filled polymers: formulation and computational aspects", Journal of the Mechanics and Physics of Solids 42(10), 1533-1550, 1994. 